\title{
Time-dependent $Z-R$ relationships for estimating rainfall fields from radar measurements
}

\author{
L. Alfieri ${ }^{1,2}$, P. Claps ${ }^{1}$, and F. Laio ${ }^{1}$ \\ ${ }^{1}$ Dipartimento di Idraulica, Trasporti ed Infrastrutture Civili (DITIC), Politecnico di Torino, Torino, Italy \\ ${ }^{2}$ Institute for Environment and Sustainability (IES), Joint Research Centre, EC, Ispra, Italy
}

Received: 26 March 2008 - Revised: 29 October 2009 - Accepted: 22 December 2009 - Published: 26 January 2010

\begin{abstract}
The operational use of weather radars has become a widespread and useful tool for estimating rainfall fields. The radar-gauge adjustment is a commonly adopted technique which allows one to reduce bias and dispersion between radar rainfall estimates and the corresponding ground measurements provided by rain gauges.

This paper investigates a new methodology for estimating radar-based rainfall fields by recalibrating at each time step the reflectivity-rainfall rate $(Z-R)$ relationship on the basis of ground measurements provided by a rain gauge network. The power-law equation for converting reflectivity measurements into rainfall rates is readjusted at each time step, by calibrating its parameters using hourly $Z-R$ pairs collected in the proximity of the considered time step. Calibration windows with duration between 1 and $24 \mathrm{~h}$ are used for estimating the parameters of the $Z-R$ relationship. A case study pertaining to 19 rainfall events occurred in the north-western Italy is considered, in an area located within $25 \mathrm{~km}$ from the radar site, with available measurements of rainfall rate at the ground and radar reflectivity aloft. Results obtained with the proposed method are compared to those of three other literature methods. Applications are described for a posteriori evaluation of rainfall fields and for real-time estimation. Results suggest that the use of a calibration window of $2-5 \mathrm{~h}$ yields the best performances, with improvements that reach the $28 \%$ of the standard error obtained by using the most accurate fixed (climatological) $Z-R$ relationship.
\end{abstract}

\section{Introduction}

Advances achieved in the recent past in radar technology and in the methods for processing data are leading to an increasing confidence toward the use of radar-based rainfall

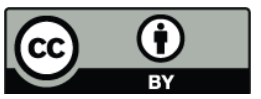

Correspondence to: L. Alfieri (lorenzo.alfieri@jrc.ec.europa.eu) estimates into hydrologic analyses and simulations. An accurate knowledge of the spatial characteristics and the amount of rainfall falling on a catchment area is of crucial importance in flood forecasting and warning systems (Smith, 1993; Claps and Siccardi, 1999; Arnaud et al., 2002; Brath et al., 2004), and can substantially improve the allocation of water resources for agricultural uses as well as for hydroelectric production (Alfieri et al., 2006). Further, an accurate quantitative precipitation estimation through radar measurements can provide an aid to the understanding of relations between point and areal rainfall (Bacchi and Ranzi, 1996) and for defining the probability distribution of annual rainfall intensity for large return periods (i.e., high rainfall rates), due to the large amount of data that the radar collects at each scan (e.g., Koistinen et al., 2006).

The procedure for evaluating radar-based rainfall fields requires (i) to define in the best possible manner the reflectivity field $(Z)$ induced by precipitation falling toward the Earth surface, and (ii) to link $\mathrm{Z}$ to an estimate of the rainfall rate $(R)$, eventually with the aid of actual precipitation measurements. Ciach and Krajewski (1999) stressed the importance to distinguish the search for a physical dependence between rainfall reflectivity and rainfall intensity in a specific precipitation system from the goal of producing the most accurate radar-based predictions of the rainfall field at the ground level. This second objective, which is the main focus of this paper, is often pursued by pairing radar measurements (aloft) with ground data provided by rain gauge networks. The adoption of a unique climatological relationship to link $Z$ and $R$ is a widespread practice for estimating rainfall fields, due to its simplicity of use and its ability to provide, on average, low-biased estimates. The literature concerning this topic reports a large number of different $Z-R$ relationships between radar reflectivity and the corresponding rainfall rate in the form of power-laws, such as those listed by Battan (1973) and Doviak and Zrnic (1984), which include the widely adopted formulations proposed by Marshall and Palmer (1948), Joss and Waldvogel (1970),

Published by Copernicus Publications on behalf of the European Geosciences Union. 
Woodley et al. (1975), among others. However, because of the considerable variability that the raindrop size distribution shows during a rainfall event, the actual $Z-R$ relation changes continuously in space and time. Therefore, the major drawback of using a unique relationship is that it cannot account for the broad variability of the true $Z-R$ relation in the presence of different types of precipitation (e.g., convective or stratiform), as well as the variations that occur within each rainfall event (e.g., see Richards and Crozier, 1983; Smith and Krajewski, 1993; Lee and Zawadzki, 2005).

Several approaches for improving the radar estimates of rainfall based on radar-gauge comparisons have been proposed in the past years (e.g., Brandes, 1975; Zawadzki, 1975; Collier et al., 1983; Austin, 1987; Ulbrich and Lee, 1999). These methods often try to reduce the estimation uncertainty by introducing additional information such as the precipitation type (e.g., convective or stratiform), the distance of the gauge from the radar, the elevation of the radar beam, and so forth (see for example Joss and Lee, 1995; Anagnostou and Krajewski, 1999a, b; Gabella and Amitai, 2000). Recent applications of real-time procedures are reported in Seo and Breidenbach (2002), Chumchean et al. (2006), Germann et al. (2006), Chiang et al. (2007).

Legates (2000) carried out a real-time calibration of the $Z-R$ power-law relationship by considering the radar-gauge pairs of the previous month characterized by non-zero rainfall, in order to account for seasonal fluctuations due to different types of precipitation and for possible drifts of the hardware calibration. It is known that the actual $Z-R$ relation is subject to variations on much shorter time scales, but it is still not clear whether an optimal calibration period for providing the best rainfall estimates is definable.

In the present paper, we propose a simple methodology for producing accurate radar-based estimates of rainfall intensity, by readjusting the coefficients of the $Z-R$ relationship continuously in time considering short calibration windows. The procedure is tested on 19 rainfall events, with no recorded snow on the ground, that occurred in the northwestern Italy between 2003 and 2006. We use reflectivity measurements from a weather radar and rainfall data from a network of 20 rain gauges located within a range of $25 \mathrm{~km}$ from the radar.

The next section describes the procedure devised for estimating rainfall fields from radar-gauge pairs. Section 3 gives some information on the case study and the data adopted; then it shows the results obtained with the two proposed techniques and a comparison with those of some literature methods. Some conclusions are reported in the final section.

\section{Methods}

The basic idea behind this work is that, for each time step $t$, one can estimate as many $Z-R$ relationships as the number of the available data pairs in the $Z-R$ plane. When a two-para- meters power-law of the form

$Z=a \cdot R^{b}$

is adopted, the coefficients $a$ and $b$ can be obtained by fitting the relation to match at least two points identified by non-zero concurrent measurements of rainfall rate and radar reflectivity. The $Z-R$ pairs can be taken either at the same spatial coordinates (i.e., the same rain gauge $j$ ) at different times (i.e., $\left\{Z_{1}=Z\left(t_{i}, j\right), R_{1}=R\left(t_{i}, j\right)\right\}$ and $\left.\left\{Z_{2}=Z\left(t_{i+1}, j\right), R_{2}=R\left(t_{i+1}, j\right)\right\}\right)$, or for the same time step $t_{i}$, considering two neighboring rain gauges (i.e., $\quad\left\{Z_{1}=Z\left(t_{i}, j\right), R_{1}=R\left(t_{i}, j\right)\right\} \quad$ and $\left.\left\{Z_{2}=Z\left(t_{i}, j+1\right), R_{2}=R\left(t_{i}, j+1\right)\right\}\right)$, where it is conventionally assumed that $j+1$ is the rain gauge closest to $j$. If the measurements were not affected by errors, for $\left(t_{i+1}-t_{i}\right)$ approaching zero (or for the distance between $j$ and $j+1$ approaching zero) the relation obtained by using only two $Z-R$ pairs would be the most suitable to convert reflectivity measurements in rainfall rates. Of course, the relation would change when moving to a different place or considering another instant in time.

The above described procedure is not practicable, due to a number of limitations which affect the problem. First, the radar reflectivity and the ground-rainfall measurements are subject to several sources of error (e.g., see Steiner et al., 1999). As a consequence, the adoption of only two $Z-R$ pairs for estimating the coefficients $a$ and $b$ would provide scarcely-reliable and highly fickle relationships. A further major source of uncertainty is due to the non-homogeneity of the volume sampled by the two instruments and to a possible temporal lag between the two measured variables (e.g., Zawadzki, 1975). The radar carries out instantaneous measurements of volume aloft, whose size varies with the distance from the radar. The rain gauges that we considered measure cumulative rainfall depths at the ground level, by sampling an area of $200 \mathrm{~cm}^{2}$ for a duration as long as the sampling time (10 min for this study).

Because of the difficulty to define $Z-R$ pairs which refer to the same volume of atmosphere sampled, rainfall measurements are usually aggregated over longer durations. Likewise, radar data are averaged over time and space, by considering a number of pixels from the reflectivity maps nearby the position of the rain gauges and a duration corresponding to the same one adopted for the rainfall data aggregation. In this work, the choice of the aggregation period is one hour in time and nine radar pixels in space (i.e. a $1.5 \times 1.5 \mathrm{~km}^{2}$ area) centered on the pixel which contains the considered rain gauge.

In order to obtain more stable and reliable $Z$ - $R$ relationships it is necessary to increase the number of $Z-R$ pairs to be considered for estimating the $a$ and $b$ coefficients. We propose to estimate a different power-law relationship for each hourly time step $t_{i}$, where the coefficients $a$ and $b$ are obtained by considering all the available $Z-R$ pairs with nonzero rainfall rate recorded in a calibration window of duration 
$d$, taken in the proximity of the selected instant $t_{i}$. As a consequence, each power-law regression is carried out on a number of $Z-R$ pairs with an upper bound $N_{\mathrm{RG}} \cdot d$, given by the number of rain gauges $N_{\mathrm{RG}}$ times the duration $d$ (in hours) of the calibration window. Different calibration windows of durations between 1 and $24 \mathrm{~h}$ are tested, and results are shown and discussed in Sect. 3.2 and 3.3. Parameters of each powerlaw relationship are calculated by minimizing the squared differences between the observed and the estimated rainfall values. The latter are obtained through

$R=10 \wedge\left(\frac{Z^{*}}{10 \hat{b}}-\frac{\log \hat{a}}{\hat{b}}\right)$,

where $\hat{a}$ and $\hat{b}$ are the coefficients to estimate. Equation (2) is obtained from Eq. (1), by considering that the reflectivity data are provided in the form $Z^{*}=10 \log Z$ [dBZ]. Note that, despite Eq. (2) represents a straight line in the bilogarithmic plane $\log \mathrm{Z}-\log R$, the regression procedure is carried out in the $Z-R$ plane by means of non-linear techniques. The adopted optimization algorithm is a subspace trust region method and is based on the interior-reflective Newton method. The optimized coefficients $\hat{a}$ and $\hat{b}$ are estimated iteratively by taking those obtained from the linear regression method (see Eq. 3) as first attempt values. The non-linear optimization causes a substantial heteroscedasticity of the estimation residuals, but it is necessary for obtaining regressions producing small errors also at high rainfall intensities, which is important when dealing with extreme precipitation events. Further, this approach has the advantage of producing almost unchanged $Z-R$ relationships when a threshold for the minimum considered value of reflectivity is set. This is better clarified at the end of this section.

In order to assess the performances of the mentioned nonlinear regression method we carried out a comparison of the results obtained with this method and with the commonly adopted method based on the equation

$\log Z=\log \hat{a}^{\prime}+\hat{b}^{\prime} \log R$

derived from Eq. (1), where $\hat{a}^{\prime}$ and $\hat{b}^{\prime}$ are the estimated values of $a$ and $b$. Results of such comparison are discussed in Sect. 3.2.

The calibration procedure was then used both for (i) a posteriori evaluation of the rainfall field, hereafter referred to as "continuous-time" (CT) readjustment, and (ii) for real-time (RT) estimation. In the first case, for each time step $t_{i}$, the $Z-R$ relationship is estimated by considering the $Z-R$ pairs for a time window of duration $d$ centered on $t_{i}$, i.e., taking all the available pairs between $t_{i}-d / 2$ and $t_{i}+d / 2$. In real-time monitoring the aim is to estimate the current rainfall field from reflectivity measurements at the same instant $t_{i}$, where each relation is estimated from the $Z-R$ pairs of the preceding hours. In this case the relation to use at the time $t_{i}$ was inferred from the $Z-R$ pairs between $t_{i-1}-d$ and $t_{i-1}$. Figure 1 shows a scheme of the calibration windows to assume

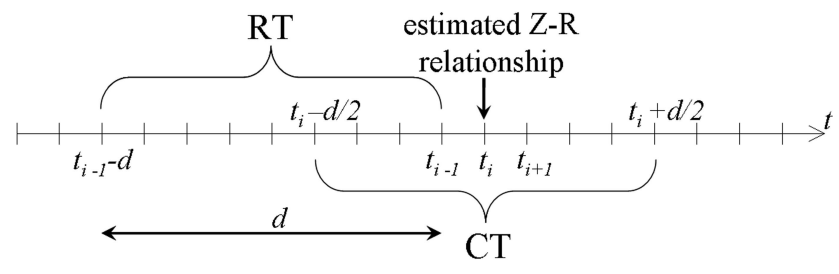

Fig. 1. Calibration windows considered for estimating the $Z-R$ relationship at a given time step $t_{i}$. Both windows for real-time (RT) estimation and continuous-time (CT) readjustment are shown.

(both for $\mathrm{CT}$ readjustment and RT estimations) for a certain duration $d$ and time step $t_{i}$, where $t$ and $d$ are expressed in hours.

Some further details are needed to clarify the operational use of the method. For some time windows no valid $Z-R$ relationship was found, either because few $Z-R$ pairs were available for the corresponding calibration window (i.e., rainfall was zero for most of the rain gauges) or because the coefficients of the power-law did not comply with the imposed constraints, $\hat{a}>1$ and $\hat{b}>1$. We rejected the estimated $Z-R$ relationships characterized by coefficients $\hat{a}<1$ or $\hat{b}<1$, in that they produce unreliable estimates of the rainfall rate for large values of the reflectivity. A physical interpretation of the assumption $\hat{b}>1$ was also suggested by Smith and Krajewski (1993), who considered the effect of the variability of the raindrop characteristics within a statistical model for estimating the power-law parameters.

For these cases the relation to adopt was chosen as the closest one in time. In particular, for CT estimation, when the calibration window $\left[t_{i}-d / 2, t_{i}+d / 2\right]$ does not provide a valid relationship, the windows $\left[t_{i+1}-d / 2, t_{i+1}+d / 2\right]$, $\left[t_{i-1}-d / 2, t_{i-1}+d / 2\right],\left[t_{i+2}-d / 2, t_{i+2}+d / 2\right],\left[t_{i-2}-d / 2\right.$, $\left.t_{i-2}+d / 2\right]$ and so forth, are tested progressively until the first relationship with valid coefficients is found. Likewise, in RT estimation, the time windows $\left[t_{i-2}, t_{i-2}-d\right],\left[t_{i-3}, t_{i-3}-d\right]$, $\left[t_{i-4}, t_{i-4}-d\right]$ are considered. The relation obtained from the bulk adjustment is used if none of these windows provides a valid result.

Another operational problem regards calibration window at the beginning or at the end of a rainfall event. A shorter temporal window is assumed for evaluating the $Z-R$ relationship in these cases, by considering only the available period. For example, if one sets a calibration window of duration $d=5 \mathrm{~h}$ in RT estimation, the reflectivity field of the first time step, $Z\left(t_{1}\right)$, will be converted into rainfall rates by using the relationship derived from the bulk adjustment. Then, for $t=t_{2}$, the method tries to calibrate a relationship by considering the $Z-R$ pairs with non-zero rainfall rate at $t_{1}(d=1 \mathrm{~h})$. For $t=t_{3}$, the calibration window will be $\left[t_{1}, t_{2}\right]$, therefore $d=2 \mathrm{~h}$. In turn, the subsequent time steps assume a duration $d=3, d=4$, and finally $d=5 \mathrm{~h}$ from $t_{6}$ onwards (i.e., for the intervals $\left[t_{1}, t_{5}\right],\left[t_{2}, t_{6}\right]$, etc.), till the last time step of the 
rainfall event. It is worth noting that the radar often measures a low but non-zero reflectivity even when no rainfall is detected from any rain gauge. Thus, if we were to apply a $Z-R$ relationship continuously for estimating rainfall rates we would obtain a weak persistent rainfall rate, spread out over the whole territory. Furthermore, if all the pairs with non-zero reflectivity and zero gauged rainfall were used for calibrating the overall $Z-R$ relationship, the subsequent rainfall estimates would turn out to be highly biased. In order to reduce this effect we carried out a further analysis, both for $\mathrm{CT}$ readjustment and RT estimation, which consists in setting a threshold $\left(Z_{\mathrm{MIN}}\right)$ for the lowest reflectivity value to be considered. Then, reflectivity values below the threshold are not considered for evaluating the $Z-R$ relationship and a zero rainfall rate is attributed to the data with $Z \leq Z_{\mathrm{MIN}}$.

The error characteristics of the estimated rainfall values are assessed by applying a cross-validation procedure for all the considered durations of the calibration window. This is carried out by excluding one rain gauge at a time from the evaluation of the $Z-R$ relationship and then comparing the estimated rainfall depth with the actual measurement at the excluded station. The quality of the estimation procedure was assessed by means of the root mean squared error (RMSE), the mean absolute error (MAE) and the estimation bias, which were calculated as follows:

$\mathrm{RMSE}=\sqrt{\frac{1}{N} \sum_{\forall t_{i}} \sum_{\forall j}\left(R_{t_{i}, j}-G_{t_{i}, j}\right)^{2}}$

$\mathrm{MAE}=\frac{1}{N} \sum_{\forall t_{i}} \sum_{\forall j}\left|R_{t_{i}, j}-G_{t_{i}, j}\right|$

$\operatorname{bias}=\frac{1}{N} \sum_{\forall t_{i}} \sum_{\forall j}\left(R_{t_{i}, j}-G_{t_{i}, j}\right)$

for all the considered durations of the calibration window. In Eqs. (4)-(6) we indicate with $G_{t_{i}, j}$ the measured hourly rainfall depth at the time $t_{i}$ and at the $j$-th rain gauge, while $R_{t_{i}, j}$ is the estimated value obtained from the corresponding radar reflectivity, by following the cross-validation procedure. The differences $\left(R_{t_{i}, j}-G_{t_{i}, j}\right)$ represent the estimation residuals, while $N$ is the number of available $Z-R$ pairs.

\section{Application and discussions}

\subsection{Case study}

The study region is a flat/hilly area located in the north-west of Italy, nearby the city of Turin, where the Regional Agency for the Protection of the Environment (ARPA Piemonte) manages a weather radar and a network of automatic rain gauges (see Fig. 2).

The radar considered in this study is a C-band Doppler and dual polarization system with a digital receiver, located at

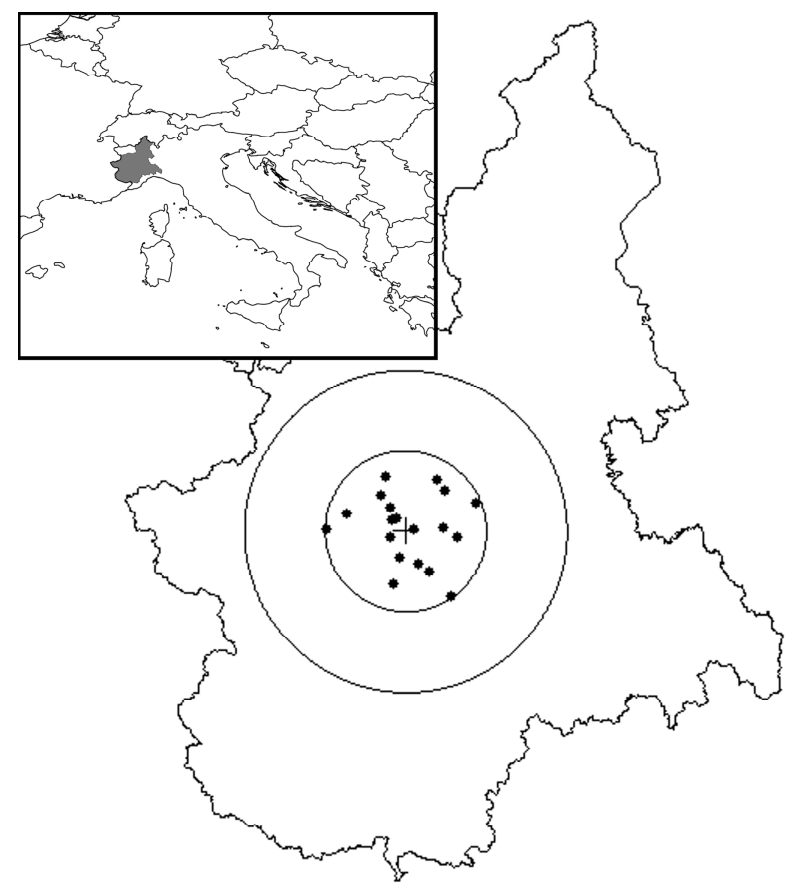

Fig. 2. Geographical setting of the Piedmont region and location of the rain gauges (black dots), the Bric della Croce radar (plus symbol) and range rings at 25 and $50 \mathrm{~km}$ from the radar.

"Bric della Croce", over the Turin hills at $736 \mathrm{~m}$ a.s.l., since 1999. ARPA Piemonte provides maps of the reflectivity factor of precipitation on a cartesian grid of 250 by $250 \mathrm{~km}$ with a resolution of $500 \mathrm{~m}$ in space and $10 \mathrm{~min}$ in time. The adopted radar product is a 2-D reflectivity map of the lowest visible radar cell with no correction for vertical profile of reflectivity, showing the reflectivity at horizontal polarization. A technique for clutter suppression is operationally implemented in the post-processing of polar volumes, which is based on three different tests to detect clutter affected data. We refer to Bechini and Cremonini (2002) and to Cremonini and Bechini (2003) for a thorough description of the considered weather radar and the processing of the collected data. Ground rainfall measurements are taken every 10 min by a network of tipping bucket rain gauges with a lower threshold of rainfall detection of $0.2 \mathrm{~mm} / 10 \mathrm{~min}$.

We selected 19 rainfall events among those with the highest daily rainfall depths between the years 2003 and 2006, which add up to a cumulated rainfall depth of $560 \mathrm{~mm}$, on average among the considered rain gauges. The event durations vary between few hours and three days. Rainfall measurements at 20 rain gauges and the corresponding maps of rainfall reflectivity were collected for the considered events. The considered rain gauges are rather uniformly distributed on the study area, at distances between 3 and $25 \mathrm{~km}$ from the radar; the reflectivity measurements over them are taken at heights below $1000 \mathrm{~m}$ from the ground. Overall, the adopted dataset counts on 10639 available hourly $Z-R$ pairs. 
Such a simple case study was intentionally chosen for emphasizing the amount of estimation uncertainty which derives from the use of a constant $Z-R$ relationship. By comparison we aim to consider a more realistic variability of the $Z-R$ relation at finer time scales, which is able to account changes in the raindrop size distribution and in the vertical velocity of air masses, among others. In addition, at farther ranges and higher beam elevations the estimation uncertainty increases, due to several sources of error such as attenuation of the radar beam, non-homogeneous beam filling, evaporation or growth of rain below the radar beam height, so that identifying the extent of each single source of error becomes increasingly difficult. By limiting the study area to a close range from the radar, some range-dependent sources of error get a reduced impact on the overall error characteristics. It is noteworthy that the proposed methodology carries some advantages also with regard to sources of error that are not range-dependent. In fact, the self-calibration properties of the $Z-R$ relationship over short time spans allow the procedure to correct for those errors that vary in time, such as attenuation due to wet radome and errors in radar calibration.

\subsection{Continuous-time (CT) readjustment}

The procedure described in Sect. 2 was first applied for the CT estimation of the hourly rainfall field by testing 24 durations of the calibration window, ranging between 1 and $24 \mathrm{~h}$.

Results are compared with those which stem from the application of the $Z-R$ relation that is currently adopted at ARPA Piemonte, $Z=300 R^{1.5}$ (Joss and Waldvogel, 1970), and with those obtained by using the power-law relation which globally minimizes the squared sum of the estimation residuals. This latter procedure consist in estimating the coefficients $\hat{a}$ and $\hat{b}$ from Eq. (2) on the whole sample of 10639 pairs, and leads to the relation $Z=79.1 R^{1.81}$, hereafter referred to as "bulk adjustment". For the CT readjustment procedure we also applied a method which evaluates a different $Z-R$ relationship for each of the 19 considered rainfall events (referred to as "event adjustment").

Figure 3a shows the RMSE of the rainfall rates estimated with the CT readjustment (thick solid line) for the considered durations, together with the RMSE obtained from the event adjustment, the bulk adjustment and the Joss and Waldvogel $(\mathrm{J}-\mathrm{W})$ relation (thick circles). The MAE and the bias of estimation are represented in Fig. 3b and c, respectively.

Figure 3 demonstrates that an improvement towards the $\mathrm{J}-\mathrm{W}$ relation is achievable by assuming a unique $Z-R$ relationship derived from a bulk adjustment carried out on all the available $Z-R$ pairs. As shown in Fig. 3c, this result is due to a substantial reduction of the estimation bias. Table 1 shows the results of a comparison between the use of a linear regression on $\log R$ as in Eq. (3) and the adoption of the analytic expression of Eq. (2). Results in Table 1 demonstrate a general reduction of the error when us-
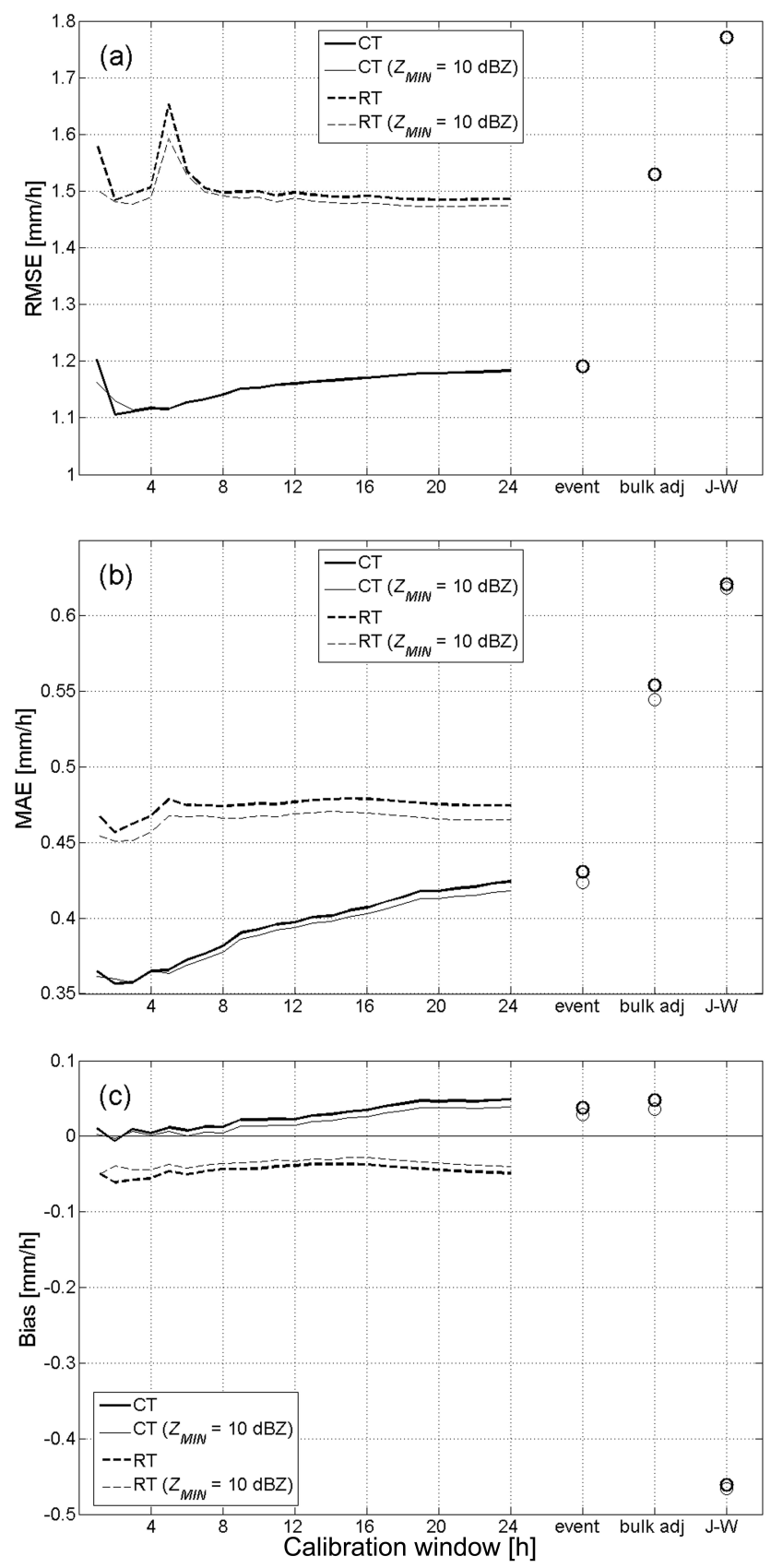

Fig. 3. RMSE (a), MAE (b) and bias of estimation (c) for different calibration windows and comparison with the results obtained with the event adjustment, bulk adjustment and the J-W relation. Continuous-time (CT) readjustment and real-time (RT) estimation approaches are shown, both evaluated by assuming a lower threshold of 0 and $10 \mathrm{dBZ}$ for the reflectivity values.

ing a non-linear fit as in Eq. (2), except for the MAE with $Z_{\mathrm{MIN}}=10 \mathrm{dBZ}$. Nevertheless, the linear regression on $\log R$ produces a considerable bias and a substantial variability of the estimated coefficients $\hat{a}$ and $\hat{b}$ with the threshold $Z_{\mathrm{MIN}}$ (see columns 2 and 3 ). The adoption of a calibrated relation 
Table 1. Error characteristics and coefficients of the power-law relationship (Eq. 1) obtained with the adoption of a unique $Z$ - $R$ relation, evaluated with the linear (Eq. 3) and the non-linear (Eq. 2) methods described in Sect. 2. The corresponding results are also reported for the case of assuming a threshold $Z_{\mathrm{MIN}}=10 \mathrm{dBZ}$.

\begin{tabular}{lcccc}
\hline & \multicolumn{2}{c}{ Linear regression (Eq. 3) } & \multicolumn{2}{c}{ Non-linear regression (Eq. 2) } \\
& $Z_{\mathrm{MIN}=0 \mathrm{dBZ}}$ & $Z_{\mathrm{MIN}}=10 \mathrm{dBZ}$ & $Z_{\mathrm{MIN}}=0 \mathrm{dBZ}$ & $Z_{\mathrm{MIN}}=10 \mathrm{dBZ}$ \\
\hline$\hat{a}$ & 106 & 137 & 79 & 78 \\
$\hat{b}$ & 2.02 & 1.64 & 1.81 & 1.82 \\
$\mathrm{RMSE}[\mathrm{mm} / \mathrm{h}]$ & 1.67 & 1.56 & 1.53 & 1.53 \\
$\mathrm{MAE}[\mathrm{mm} / \mathrm{h}]$ & 0.57 & 0.53 & 0.55 & 0.54 \\
$\operatorname{Bias}[\mathrm{mm} / \mathrm{h}]$ & -0.21 & -0.16 & 0.05 & 0.04 \\
\hline
\end{tabular}

for each rainfall event allows one to obtain a further considerable reduction of both RMSE and MAE. This improvement is not due to a significantly lower bias but probably to the ability to adapt the coefficients $a$ and $b$ to account for the rainfall type (e.g., convective or stratiform precipitation), as well as event-to-event differences in radar calibration, residual clutter, attenuation due to wet radome and to heavy rainfall .

The "within event" CT readjustment of the $Z-R$ relationship produces a further improvement of the estimation procedure, which is maximum for calibration windows of $2 \mathrm{~h}$, where the RMSE becomes the $28 \%$ lower than in the case of bulk adjustment. Figure 3 a denotes a progressive reduction of the RMSE as the width of the calibration window narrows. The sudden increase of the RMSE that occurs for a calibration window of one hour is due to the instability of some obtained relationships, which are evaluated on a limited number of $Z-R$ pairs. On the contrary, long calibration windows generate more stable fits, because they are estimated on larger sets of $Z-R$ pairs, but as expected the corresponding $Z-R$ relation turns out to be less accurate.

The robustness of the obtained results is confirmed in Fig. 4, which shows that the qualitative behavior of the RMSE as a function of $d$, as shown in Fig. 3a, is retained even when considering only the estimation residuals of above-threshold rainfall rates, with thresholds varying between 1 and $10 \mathrm{~mm} \mathrm{~h}^{-1}$. Figure 4 shows the dimensionless ratios between the RMSE of the above threshold rainfall estimates and the corresponding (i.e., considering the same thresholds) values obtained with the $\mathrm{J}-\mathrm{W}$ method. Note that the best improvements of the CT method toward the J-W method occur for $Z-R$ pairs with thresholds between 3 and $5 \mathrm{~mm} \mathrm{~h}^{-1}$.

The whole procedure was then repeated by setting a reflectivity threshold $\left(Z_{\mathrm{MIN}}\right)$, as described in Sect. 2, and the corresponding results are shown in Fig. 3a, b, and c with a thin solid line and thin circles. We found that $Z_{\mathrm{MIN}}=10 \mathrm{dBZ}$ is a reasonable threshold value to adopt, which corresponds to about $0.3 \mathrm{~mm} \mathrm{~h}^{-1}$ for the relation indicated above in this section, obtained from the bulk adjustment method (i.e., with $\hat{a}=79.1$ and $\hat{b}=1.81$ ). Such value was chosen to minimize

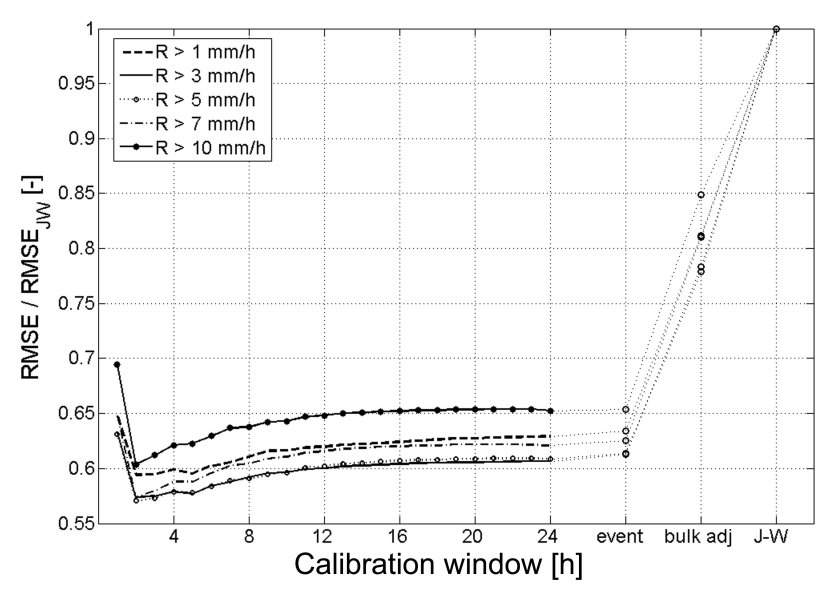

Fig. 4. Ratios between the RMSEs obtained by the continuoustime (CT) readjustment, event adjustment, bulk adjustment, J-W relation and the corresponding RMSEs derived by the use of the J$\mathrm{W}$ relation. Results are plotted by considering rainfall rates above different thresholds between 1 and $10 \mathrm{~mm} \mathrm{~h}^{-1}$.

the resulting estimation error and therefore improving the removal of non-meteorological echoes. Results that stem from this method are slightly better than those obtained in the case $Z_{\mathrm{MIN}}=0 \mathrm{dBZ}$, except for a calibration window of $2 \mathrm{~h}$. This discontinuity is due to the instability of some estimated $Z-R$ relationships, which in this case occurs also for calibration windows as short as $2 \mathrm{~h}$. In fact, the introduction of a threshold on $Z$ reduces the number of $Z-R$ pairs considered in each regression. On the other hand, the use of a threshold has the advantage to prevent the estimation of $Z-R$ relationships considering only very low values of $Z$, which may produce large errors when used to convert high reflectivities into rainfall rates.

\subsection{Real-time (RT) estimation}

Similarly to the CT procedure, the RT estimation was carried out for durations of the calibration window between 1 and $24 \mathrm{~h}$, again for the two cases of $Z_{\mathrm{MIN}}=0 \mathrm{dBZ}$ and $Z_{\mathrm{MIN}}=10 \mathrm{dBZ}$. 
The RT estimation is carried out in cross-validation mode for all the rainfall events, and the results are represented in Fig. 3a, b, and $\mathrm{c}$ with a thick dashed line $\left(Z_{\mathrm{MIN}}=0 \mathrm{dBZ}\right)$ and a thin dashed line $\left(Z_{\mathrm{MIN}}=10 \mathrm{dBZ}\right)$. In this case, results are compared to those of the J-W relation and of the bulk adjustment. The event adjustment is not a viable method in the real-time estimation, so it will not be considered explicitly (in Fig. 3) when comparing the different approaches.

As expected, the RT method estimates turn out to be less accurate than the CT estimates, with a slight underestimation, on average, for all the considered durations of the calibration window (see Fig. 3c). Although the estimation errors are, on average, lower than those of the bulk adjustment method (see Fig. 3b), the RMSE is higher for durations of the calibration window of 1,5 , and $6 \mathrm{~h}$. In particular, the anomalous peak for $d=5 \mathrm{~h}$ (Fig. 3a) is due to a single large error, associated to a point with high reflectivity (about $39 \mathrm{dBZ}$ ) and no gauged rainfall on the ground, whereas the $Z-R$ relationship provides a very high rainfall rate estimate $\left(69 \mathrm{~mm} \mathrm{~h}^{-1}\right)$.

The introduction of the threshold $Z_{\mathrm{MIN}}=10 \mathrm{dBZ}$ induces an improvement of the overall performances of the RT estimation, in that the corresponding bias, the RMSE and the MAE are all reduced and the peaks of the RMSE are reduced as well. The comparison in Fig. 3, between the RT and CT methods, suggests that the most significant reduction of the estimation error is given by considering the $Z-R$ pairs at the present time $t_{i}$, for calibrating the analytic relationship. This is clearly shown in the comparison of both the RMSE and MAE, between the CT and RT methods with a calibration window of one hour. In this case the procedure applied by the two methods is the same, apart from the time step considered for calibrating the $Z-R$ relationship, which is $t_{i}$ for the CT method and $t_{i-1}$ for the RT method.

It is worth noting that the two proposed methods allow one to obtain a considerable reduction of the mean absolute error (see Fig. 3b) if compared with the corresponding most accurate literature approaches that were tested in this work. In fact, the CT readjustment with $d=3 \mathrm{~h}$ produces a MAE which is about $15 \%$ lower than for the event adjustment, while the MAE of the RT estimation with $d=24 \mathrm{~h}$ is roughly $14 \%$ lower than in the case of using a single climatological relationship (i.e., bulk adjustment). Differently, the corresponding reduction in the RMSE (Fig. 3a) is lower (6\% for the CT readjustment and $4 \%$ for the RT estimation). This suggests that few largest estimation errors are retained and largely affect the RMSE, which depends on a squared measure of residuals. A possible explanation to this outcome is that large errors are not removed because they are not ascribable to the non-identification of a suitable $Z-R$ relationship, but rather to other sources of error which affect the radar measurement (see Steiner et al., 1999), and particularly to observations affected by ground clutter contamination.

We plotted in Fig. 5 the empirical frequency distributions $f(a)$ and $f(b)$ of the coefficients $a$ and $b$ of the estimated power-laws, for the two proposed methodologies. We (a) Continuous-Time $(d=3 \mathrm{~h})$
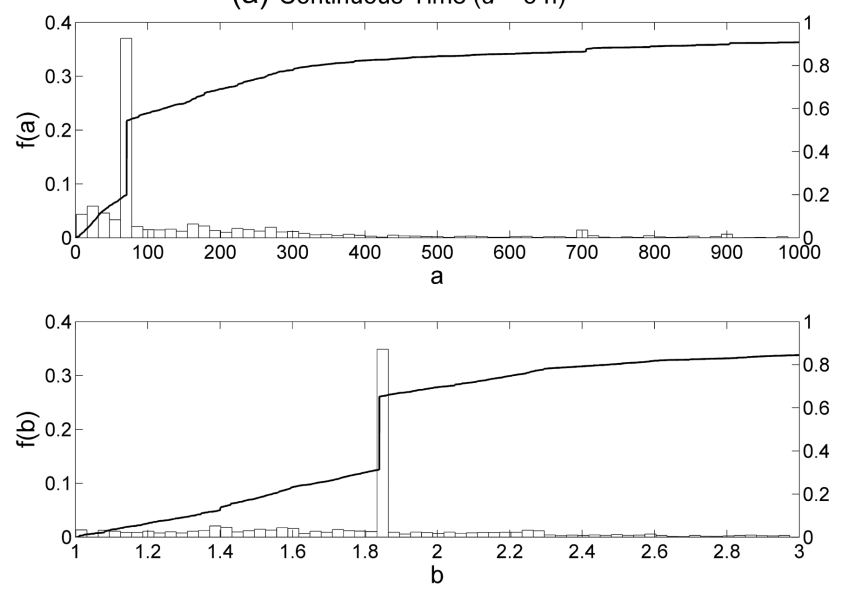

(b) Real-Time $(d=24 \mathrm{~h})$
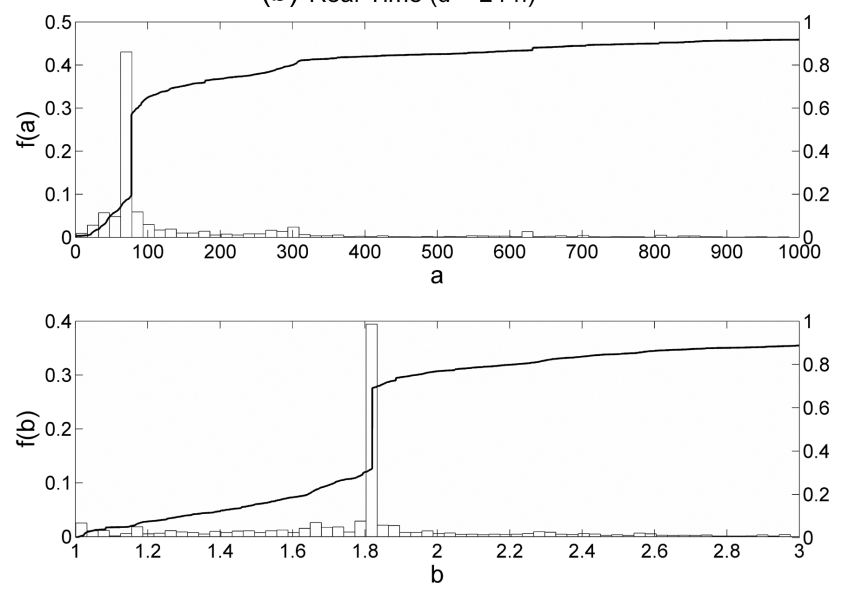

Fig. 5. Empirical frequency distribution (bar chart) and cumulative distribution (solid line) of the estimated coefficients $a$ and $b$ of the $Z-R$ power law. Top panels (a): continuous-time $(\mathrm{CT})$ readjustment $(d=3 \mathrm{~h})$; bottom panels $(\mathbf{b})$ : real-time $(\mathrm{RT})$ estimation $(d=24 \mathrm{~h})$.

considered a calibration window of $3 \mathrm{~h}$ for the CT method (Fig. 5a), and of $24 \mathrm{~h}$ for the RT method (Fig. 5b). The corresponding cumulative distributions are also represented with a continuous solid line. The spread of the estimated coefficients is clearly shown from the four panels of the figure. Further, one can note the sudden jump of the cumulative distributions for the two coefficients assuming values $\hat{a}=79.1$ and $\hat{b}=1.81$. These represent the frequencies of rejected regressions, and amount in both cases to about $35-40 \%$ of the number of estimated values. Operationally, they are replaced with the coefficients of the bulk adjustment regression indicated above.

In Fig. 6 we represented a comparison between the rainrate measured at a rain gauge during an event (white bars) and the corresponding estimates obtained with the methods described in this paper, by assuming the threshold $Z_{\mathrm{MIN}}=10 \mathrm{dBZ}$ for all the cases. Again, calibration windows of 3 and $24 \mathrm{~h}$ are considered respectively for CT and RT 


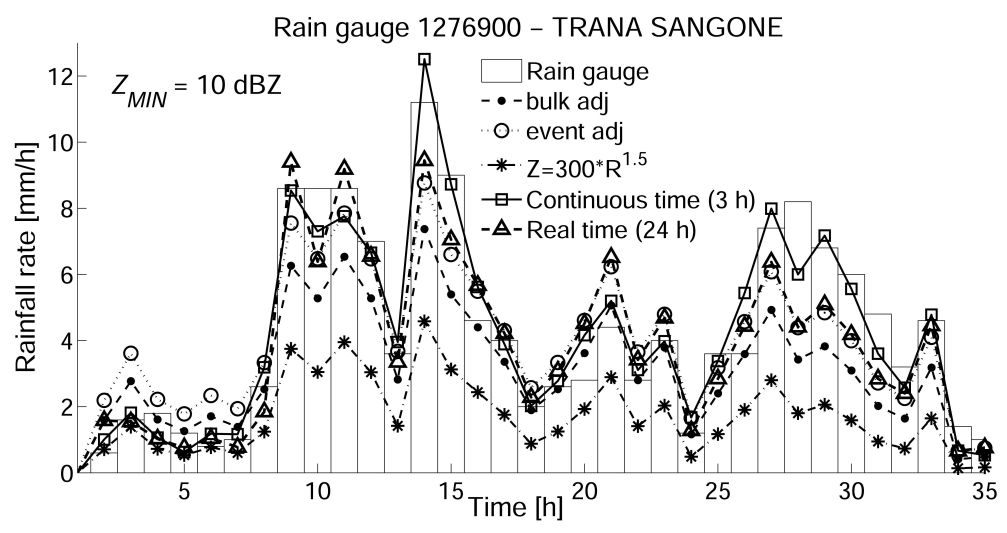

Fig. 6. Comparison of rainfall rates measured at a rain gauge and the corresponding estimates for one of the rainfall events, by using the bulk adjustment method, the event adjustment, the J-W relation, the continuous-time (CT) readjustment and the real-time (RT) estimation.

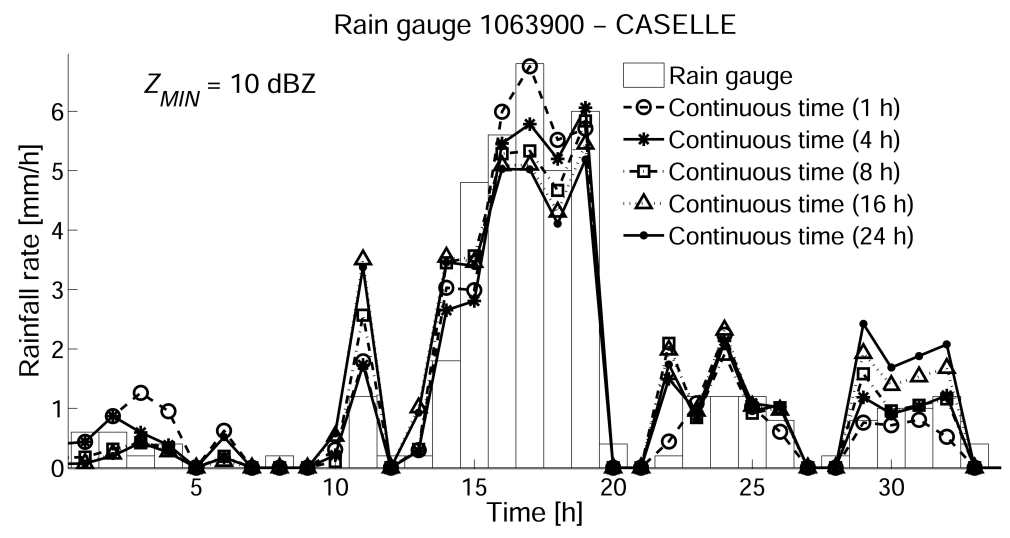

Fig. 7. Comparison of rainfall rates measured at a rain gauge and the corresponding estimates for one of the rainfall events, by using the continuous-time (CT) readjustment with five different calibration windows, between 1 and $24 \mathrm{~h}$.

methods. Figure 6 clearly shows the ability of the CT method to accurately estimate rainfall rates, while the J-W method always provides a considerable underestimation. This picture is representative of the typical behavior of the tested methodologies. It is shown for giving a more direct way for comparing the different estimation performances, while one should refer to Fig. 3 for a more objective statistical evaluation. Similarly, Fig. 7 shows a comparison between the gauged rainfall during a selected event and the estimated values obtained by testing the CT method with five different calibration windows between 1 and $24 \mathrm{~h}$. One can note that the five procedures generally provide reliable estimates, especially for short durations of the calibration window. Indeed, even the 1-h time window provides good results. These findings are of crucial importance in that, although the $\mathrm{CT}$ procedure is an a posteriori analysis of rainfall rates, by waiting just $1 \mathrm{~h}$ from a given radar measurement, the accuracy of estimation substantially improves (compared to the corresponding real-time estimates). This means that the CT method with a 1-h time window can be considered a valid "near real-time" alterna- tive for estimation, with important implications for hydrological applications, where a 1 -h lag time is a viable compromise in place of substantial quantitative improvements.

Finally, a further set of graphs is reported in Fig. 8, which shows the scatter plots between measured and estimated rainfall rates for the five methods described in this work together with their corresponding RMSE, both in linear (left side) and logarithmic (right side) scale. Again, the selected durations of the calibration windows are $3 \mathrm{~h}$ for the CT method and $24 \mathrm{~h}$ for the RT method. Furthermore, all the scatter plots reported in Fig. 8 are obtained by setting the threshold $Z_{\mathrm{MIN}}=10 \mathrm{dBZ}$ as explained throughout this article, in that the resulting error characteristics are slightly better than those provided by the case of threshold $Z_{\mathrm{MIN}}=0 \mathrm{dBZ}$. One can note, in the right side panels of Fig. 8 , the value of $0.2 \mathrm{~mm} \mathrm{~h}^{-1}$ as the rain gauge instrumental resolution. Such graphical representation confirms the usefulness of setting a threshold for very low values of $Z$ and of the use of a non-linear regression as in Eq. (2), which gives larger weights to high reflectivity values. 

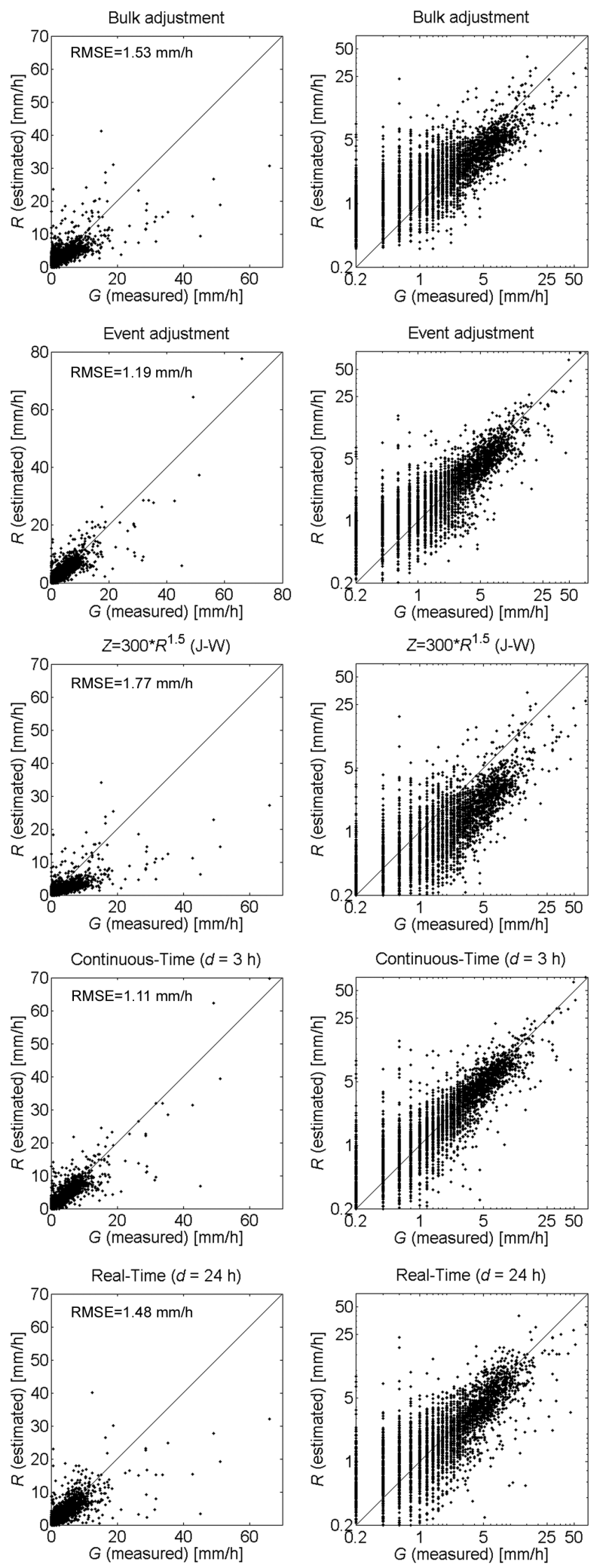

Fig. 8. Scatter plots between measured and estimated rainfall rates for the five methods described in this article, both in linear (left side) and logarithmic (right side) scale. The 1:1 line is also shown in each plot. For all the five methods the threshold $Z_{\mathrm{MIN}}=10 \mathrm{dBZ}$ is adopted.

\section{Conclusions}

This paper presents a simple procedure for using $Z-R$ relationships continuously updated in time, useful both for reanalysis of rainfall fields and for real time estimation, and carries out a comparison of the overall performances by testing different calibration windows. The outcomes of these methods are also compared with those of three other calibration methods reported in the literature. The adopted procedure aims at producing the most accurate radar-based rainfall estimates and we do not claim any physical interpretations of the obtained relationships. The estimated coefficients $\hat{a}$ and $\hat{b}$ of the power-law relationships as in Eq. (1) are bounded only to prevent instability problems to occur. As a result, they are considerably spread out around those of the mean $Z-R$ relation derived from the bulk adjustment method, and often assume different values from those reported in the literature. The obtained coefficients $\hat{a}$ and $\hat{b}$ include the effect of sampling errors of the radar measurements and the uncertainty which derives from coupling reflectivity measurements aloft with ground rainfall rates measured by the rain gauges.

Results are promising, as both the continuous-time (CT) and the real-time (RT) approaches demonstrate substantial improvements compared to the other tested methods, especially when a threshold for the minimum reflectivity to consider is adopted. In particular, we suggest a calibration window of $3 \mathrm{~h}$ for the $Z-R$ relationship when applying the continuous-time (CT) readjustment. In real-time (RT) estimation, a calibration window between roughly 8 and $24 \mathrm{~h}$ is a reasonable choice, which provides good accuracy of estimation and is not affected by instability problems. Even though these results refer specifically to the case analyzed in this study, they are rather robust, since are based on more than $10^{4}$ estimated hourly values of precipitation.

The strength of this methodology is the simplicity and the objectiveness of use. Besides, it is almost unaffected by drifts of the radar hardware calibration, due to the short time windows used for calibrating the $Z-R$ relationship. Further refinements to the proposed methodology are practicable, such as the use of multiple regressions for the rainfall estimation, which consider more than a single variable among those measured by the polarimetric radar, the removal of anomalous points from the $Z-R$ calibration (e.g., points with high reflectivity and low rain rate or vice versa), additional quality control procedures (see for example Steiner et al., 1999; Gabella and Amitai, 2000), or further thresholds for bounding the maximum values of $Z$ and $R$. Future developments of this work will be addressed at increasing the number of rainfall events and of rain gauges to consider. Farther ranges from the radar and different areal densities of the gauge network are likely to affect the space-time variability of the powerlaw coefficients and in turn the overall accuracy of the radarbased rainfall estimates. 
Acknowledgements. The authors wish to thank ARPA Piemonte for providing the data and in particular S. Barbero, R. Bechini, V. Campana, R. Cremonini, D. Rabuffetti and L. Tomassone for useful discussions on this topic. The financial support of the Italian Ministry of Education and Research (grant no. 2005080287 and 2006089189) is also acknowledged.

Edited by: A. Mugnai

Reviewed by: three anonymous referees

\section{References}

Alfieri, L., Perona, P., and Burlando, P.: Optimal water allocation for an alpine hydropower system under changing scenarios, Water Resour. Manag., 20, 761-778, 2006.

Anagnostou, E. N. and Krajewski, W. F.: Real-time radar rainfall estimation. Part I: Algorithm formulation, J. Atmos. Ocean. Tech., 16, 189-197, 1999a.

Anagnostou, E. N. and Krajewski, W. F.: Real-time radar rainfall estimation. Part II: Case study, J. Atmos. Ocean. Tech., 16, 198205, 1999b.

Arnaud, P., Bouvier, C., Cisneros, L., and Dominguez, R.: Influence of rainfall spatial variability on flood prediction, J. Hydrol., 260, 216-230, 2002.

Austin, P. M.: Relation between measured radar reflectivity and surface rainfall, Mon. Weather Rev., 115, 1053-1071, 1987.

Bacchi, B. and Ranzi, R.: On the derivation of the areal reduction factor of storms, Atmos. Res., 42, 123-135, 1996.

Battan, L. J.: Radar observations of the atmosphere, The University of Chicago Press, 1973.

Bechini, R. and Cremonini, R.: The weather radar system of northwestern Italy: an advanced tool for meteorological surveillance, in: Proceedings of the Second European Conference on Radar in Meteorology and Hydrology, Delft, The Netherlands, 400-404, 18-22 November 2002.

Brandes, E. A.: Optimizing rainfall estimates with aid of radar, J. Appl. Meteorol., 14, 1339-1345, 1975.

Brath, A., Montanari, A., and Toth, E.: Analysis of the effects of different scenarios of historical data availability on the calibration of a spatially-distributed hydrological model, J. Hydrol., 291, 232-253, 2004.

Chiang, Y. M., Chang, F. J., Jou, B. J. D., and Lin, P. F.: Dynamic ANN for precipitation estimation and forecasting from radar observations, J. Hydrol., 334, 250-261, 2007.

Chumchean, S., Seed, A., and Sharma, A.: Correcting of real-time radar rainfall bias using a Kalman filtering approach, J. Hydrol., 317, 123-137, 2006.

Ciach, G. J. and Krajewski, W. F.: Radar-rain gauge comparisons under observational uncertainties, J. Appl. Meteorol., 38, 15191525, 1999.

Claps, P. and Siccardi, F.: Mediterranean Storms, BIOS, Cosenza, 1999.

Collier, C. G., Larke, P. R., and May, B. R.: A weather radar correction procedure for real-time estimation of surface rainfall, Q. J. Roy. Meteor. Soc., 109, 589-608, 1983.
Cremonini, R. and Bechini, R.: Rainfall estimation in north-western Italy, using two polarimetric C-band radars and a dense real-time gauge network, 3rd GPM Workshop, ESTEC, Noordwijk, The Netherlands, 2003.

Doviak, R. J. and Zrnic, D. S.: Doppler radar and weather observations, Academic Press, New York, 1984.

Gabella, M. and Amitai, E.: Radar rainfall estimates in an alpine environment using different gage adjustment techniques, Phys. Chem. Earth Pt. B, 25, 927-931, 2000.

Germann, U., Galli, G., Boscacci, M., and Bolliger, M.: Radar precipitation measurement in a mountainous region, Q. J. Roy. Meteor. Soc., 132, 1669-1692, 2006.

Joss, J. and Lee, R.: The application of radar-gauge comparisons to operational precipitation profile corrections, J. Appl. Meteorol., 34, 2612-2630, 1995.

Joss, J. and Waldvogel, A.: A method to improve the accuracy of radar-measured amounts of precipitation, in: Preprints, 14th Radar Meteorology Conf., Tucson, AZ, 237-238, 1970.

Koistinen, J., Kuitunen, T., and Inkinen, M.: Area-intensity probability distributions of rainfall based on a large sample of radar data, in: Proceedings of the Fourth European Conference on Radar in Meteorology and Hydrology, Barcelona, Spain, 406409, 18-22 September 2006.

Lee, G. W. and Zawadzki I.: Variability of drop size distributions: time-scale dependence of the variability and its effects on rain estimation, J. Appl. Meteorol., 44, 241-255, 2005.

Legates, D. R.: Real-time calibration of radar precipitation estimates, Prof. Geogr., 52, 235-246, 2000.

Marshall, J. M. and Palmer W. M. K.: The distribution of raindrops with size, J. Appl. Meteorol., 5, 165-166, 1948.

Richards, W. G. and Crozier C. L.: Precipitation measurement with a C-band weather radar in Southern Ontario, Atmos. Ocean, 21, 2505-2514, 1983.

Seo, D. J. and Breidenbach, J. P.: Real-time correction of spatially nonuniform bias in radar rainfall data using rain gauge measurements, J. Hydrometeorol., 3, 93-111, 2002.

Smith, J. A.: Precipitation, in: Handbook of Hydrology, edited by: Maidment, D. R., McGraw-Hill, Inc, 1993.

Smith, J. A. and Krajewski, W. F.: A modeling study of rainfall rate reflectivity relationships, Water Resour. Res., 29, 2505-2514, 1993.

Steiner, M., Smith, J. A., Burges, S. J., Alonso, C. V., and Darden, R. W.: Effect of bias adjustment and rain gauge data quality control on radar rainfall estimation, Water Resour. Res., 35, 2487-2503, 1999.

Ulbrich, C. W. and Lee, L. G.: Rainfall measurement error by WSR$88 \mathrm{D}$ radars due to variations in $Z-R$ law parameters and the radar constant, J. Atmos. Ocean. Tech., 16, 1017-1024, 1999.

Woodley, W. L., Olsen, A. R., Herndon, A., and Wiggert, V.: Comparison of gauge and radar methods of convective rain measurement, J. Appl. Meteorol., 14, 909-928, 1975.

Zawadzki, I.: Radar-raingage comparison, J. Appl. Meteorol., 14, 1430-1436, 1975. 